\title{
A Comparative Study on the Technologies of the removal of Acetylsalicylic Acid from Water
}

\section{REKHAPALLI SRI SHREYA ${ }^{1}$ and GEETHA KANNAN ${ }^{2}$}

'Department of Chemical Engineering, Dubai Campus, Birla Institute of Technology and Science Pilani, India. ${ }^{2}$ Department of General Sciences, Dubai Campus, Birla Institute of Technology and Science Pilani India. ${ }^{*}$ Corresponding author E-mail: geetha@ dubai.bits-pilani.ac.in, rsshreya@yahoo.co.in

\author{
http://dx.doi.org/10.13005/ojc/330660
}

(Received: June 28, 2017; Accepted: July 22, 2017)

\begin{abstract}
Over the recent past, there has been a growing concern regarding the sources and effects of pharmaceutical products in the water bodies. Improper disposal of these medications have resulted in their accumulation in the environment and has eventually led to them contaminating the waters. The most common pollutant is aspirin and in this study, comparative treatment methods of aspirin in water were performed to identify the most suitable, simple and cost effective quantitative method for its removal. The treatability of aspirin by Ozonation, Aeration, Adsorption, and Photo catalytic degradation were studied in batch processes and parameters influencing the process were optimized.
\end{abstract}

Keywords: Aspirin, Salicylic acid, Treatment methods, Pharmaceuticals, pollution, Aeration, adsorption, Ozonation, Photocatalysis

\section{INTRODUCTION}

Over the past two to three decades, there has been a huge growth in the Global Pharmaceutical Market and a tremendous rise in the production of pharmaceuticals, owing to better health care facilities and medical advancements. A large number of drugs are 'over-the-counter' to which the general public has easy access. Excessive buying of medications, their improper disposal and general ignorance has led to their contamination in the environment. In a study done by the US Geological Survey, one of more of 95 chemicals tested for, which included but were not limited to pharmaceuticals and insect repellants, were found in $80 \%$ of streams samples. ${ }^{1}$
All such drugs and their residues find their way into the water bodies and ultimately pose a threat to the organisms living in there and the ecosystem containing those water systems, and even show a potential threat to humans. ${ }^{1}$

A lack of understanding and experience over the decades has contributed to the ecotoxicity already present in the environment, and the past few years have exhibited a dangerous trend in how these pollutants affect the environment in which they are present. Several classes of drugs have been detected in recent years in different regions and water bodies ${ }^{2}$. These contaminants have found their way into our drinking water as well. Statistical data has shown the most commonly found 
pharmaceutical contaminants in the environment to be non-steroidal anti-inflammatory drugs (NSAIDs) and amongst this class, acetylsalicylic acid (aspirin) is a regular pollutant ${ }^{3}$. The presence of NSAIDs in the environment has caused a damaging impact on other species. In fish and birds, NSAIDs have led to renal failure, poor egg shell quality, and imbalance in the sex ratios of certain fish $^{4}$. Most of these pharmaceutical pollutants originate from households, hospitals and nursing homes, and the effluents of drug manufacturing industries mainly due to improper disposal. ${ }^{5}$ Previous research consists of treatment methods for the removal of antibiotics and anti-depressants from wastewater. Amongst NSAIDs, studies have been conducted on the purifying waters containing ibuprofen, diclofenac, carbamazapine ${ }^{6}$ and naproxen ${ }^{7}$. The removal technologies of acetylsalicylic acid from water are still under study. Research includes, removal of aspirin by advanced membrane technology, activated charcoal and clay micelles complex ${ }^{4}$ and mechanism of acetylsalicylic aicd degradation under solar light in the presence of titanium dioxide polymeric film photocatalyst ${ }^{8}$. Further availability of information is limited as this is a recent and ongoing study.

\section{Experimental Analysis}

Pure aspirin was prepared in the laboratory and UV-Spectrophotometry was used for the estimation of aspirin and the calibration studies. A standard stock solution of aspirin of $1600 \mathrm{ppm}$ was used for all the studies. UV-Spectrophotometry method obeyed the Beer Lambert Law in the concentration range of $0-128 \mathrm{ppm}$ at $267 \mathrm{~nm}$. The detection limit and molar absorptivity were found to be 6 ppm and $922.22 \mathrm{~L} \mathrm{~mol}^{-1} \mathrm{~cm}^{-1}$ respectively.
Table. 1: Calibration of Aspirin using UV-Spectrophotometry

\begin{tabular}{lcc}
\hline S.No & $\begin{array}{c}\text { Concentration } \\
(\mu \mathrm{g} / 5 \mathrm{~mL})\end{array}$ & $\begin{array}{c}\text { Absorbance } \\
(\mathrm{Abs})\end{array}$ \\
\hline 1 & 32 & $0.172 \pm 0.005$ \\
2 & 64 & $0.332 \pm 0.004$ \\
3 & 96 & $0.488 \pm 0.022$ \\
4 & 128 & $0.657 \pm 0.017$ \\
\hline
\end{tabular}

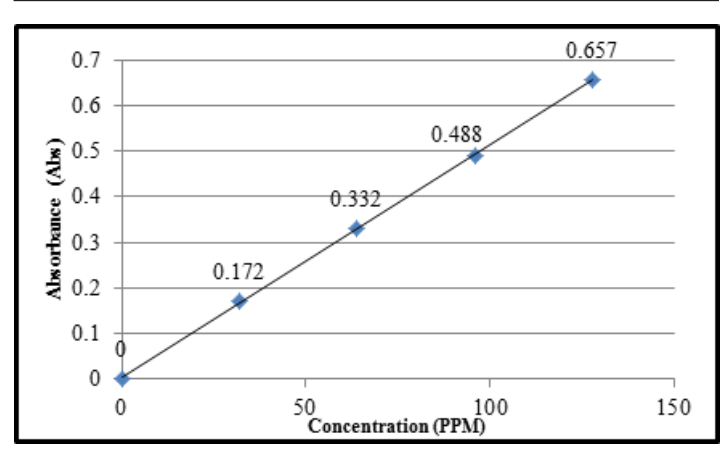

Fig.1. Calibration Curve for Aspirin by UVSpectrophotometry

Treatment Methods for the removal / transformation /degradation of Acetylsalicylic Acid:

The characteristics of acetylsalicylic acid must be analyzed in order to decide on what treatment methods are suitable for its removal from water ${ }^{9}$. The following data provides information on what kind of treatment methods would be useful and appropriate as far as aspirin is concerned. Of the many treatment methods practiced in waste water treatment plants, four were found to be theoretically suitable for this comparative study on the treatment of aspirin, namely: Ozonation, Batch Adsorption, Aeration and Photocatalysis.

Table. 2: Theoretically Suitable Methods of Treatment for Aspirin based on its Characteristics

\begin{tabular}{llll}
\hline $\begin{array}{l}\text { Method of } \\
\text { Treatment }\end{array}$ & Parameter & $\begin{array}{l}\text { Characteristics of } \\
\text { Aspirin }\end{array}$ & Considerability \\
\hline Absorption & $\begin{array}{l}\text { Log Kow } \\
\text { Hydrophobic }\end{array}$ & $\begin{array}{l}\text { Log Kow }=1.426 \\
\text { Hydrophilic }\end{array}$ & Not considered \\
Adsorption & Electrostatic Interactions & $\begin{array}{l}\text { Releases negative ions in } \\
\text { aqueous medium. }\end{array}$ & Considered \\
Ozonation & $\begin{array}{l}\text { Reacts with aromatic compounds } \\
\text { with high electron densities }\end{array}$ & $\begin{array}{l}\text { Aromatic compound. } \\
\text { Has high electron density in } \\
\text { ortho position due to electron } \\
\text { withdrawing group. }\end{array}$ & Considered \\
& $\begin{array}{l}\text { Can be mineralized to form } \\
\mathrm{CO}_{2} \text { and } \mathrm{H}_{2} \mathrm{O}\end{array}$ & Considered \\
Photocatalysis & Biodegradability and & & \\
\hline
\end{tabular}




\section{Aeration:}

The effect of aeration was studied under the influence of three different conditions:

\section{Aerial Oxidation under Ambient Conditions:}

A known volume and concentration of acetylsalicylic acid solution was taken in a $250 \mathrm{~mL}$ beaker and kept aside for analysis of the effect of simple aerial oxidation under ambient conditions. The results were summarized in Fig.2 \& Figure.3.

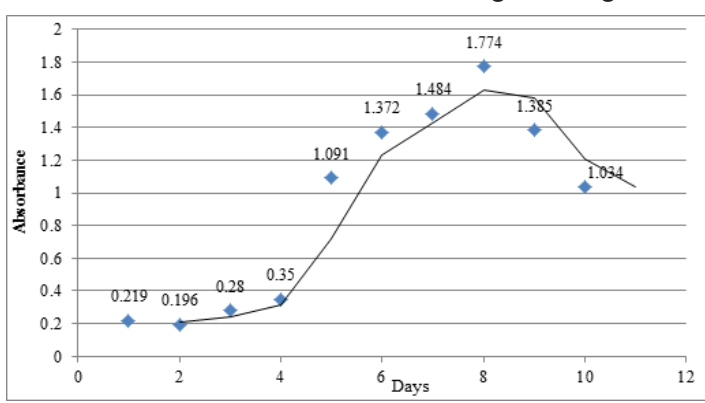

Fig. 2. Effect of Aerial Oxidation (under ambient conditions) on Acetylsalicylic Acid

\section{Aeration using an Air Pump:}

A sample solution of aspirin of known volume and concentration $(50 \mathrm{~mL})$ was taken in a beaker. Air was bubbled through the solution using an air pump. An hourly analysis was made for a span of $8 \mathrm{~h}$ and the results were summarized in Fig. 4 \& Figure.5.

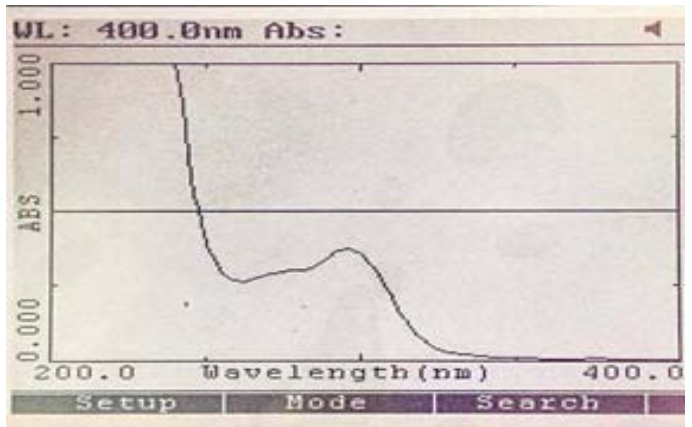

Aerial Oxidation under the influence of Increasing Temperature Ranges:

The effect of increasing temperature ranges during aerial oxidation was studied for six acetylsalicylic acid samples of known volume and concentration. Each sample was kept for $20 \mathrm{~min}$. in a water bath that was maintained at a certain range of temperature and the whole process lasted for a total of 2 hours. The results were summarized in Fig.6 \& Figure.7.

\section{Ozonation:}

Sample solutions of known volume and concentration containing acetylsalicylic acid were treated by the passage of ozone through them for 10 minutes. The results were summarized in Table. 3

\section{Adsorption:}

For adsorption, various samples of aspirin solution were tested and the parameters involved were varied. $\mathrm{pH}$ was varied from 4 to 7 , weight of carbon from $0.03 \mathrm{~g}-0.1 \mathrm{~g}$ and time from 1-4 hours. All experiments were performed in a triplicate manner to determine the reliability of the method and the optimal results were chosen. $\mathrm{A} \mathrm{pH}$ of 4 , carbon weight of $0.05 \mathrm{~g}$ and time duration of $2 \mathrm{~h}$ showed the best results. For PAC and GAC that were commercial carbons, the $\mathrm{pH}$ was altered to 4

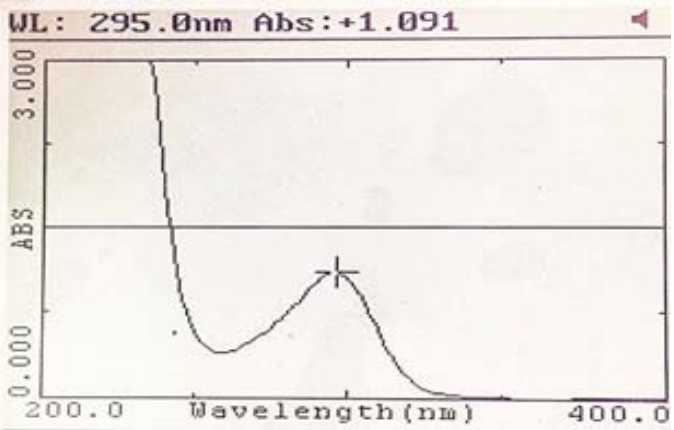

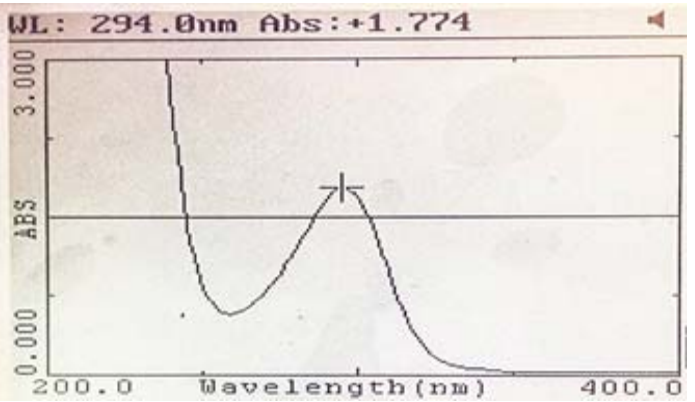

Fig. 3. UV Graphs - 'Ambient Condition' conversion of aspirin (left peak) into salicylic acid (right peak) 


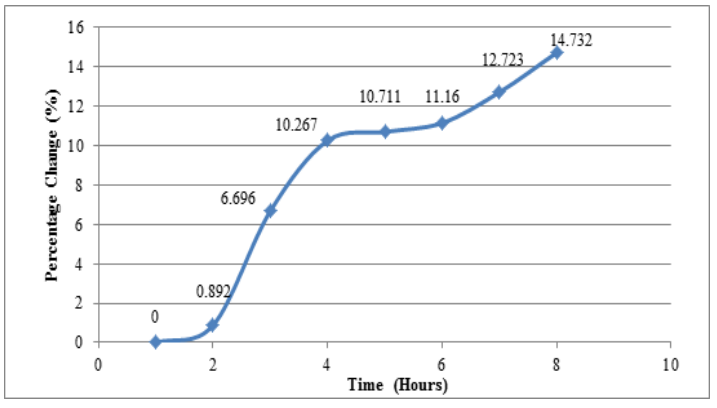

Fig. 4. Aeration Treatment using Pump

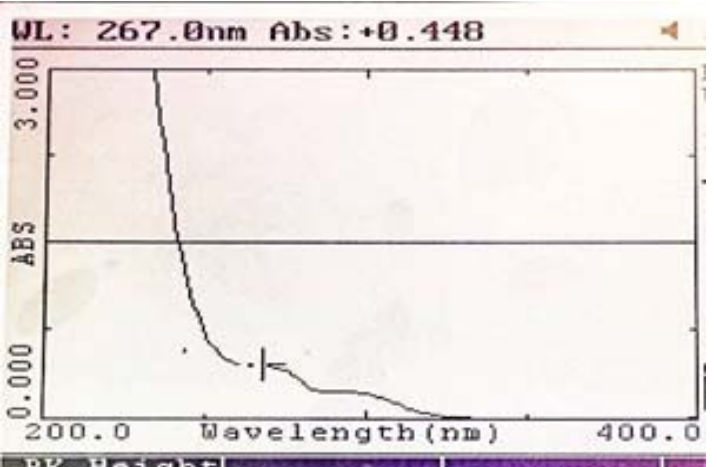

using an acetate buffer. For the date seed carbons produced in acidic and basic media, $\mathrm{pH}$ was not altered in order to study the adsorptive capacity of the carbons of such media. Batch adsorption was carried out for all four types of carbons for $2 \mathrm{~h}$ and their optimal results were measured Figure.8.

\section{Photocatalysis:}

A known volume and concentration of a sample solution of acetylsalicylic acid was taken (1600 ppm) in a beaker. Titanium dioxide was used

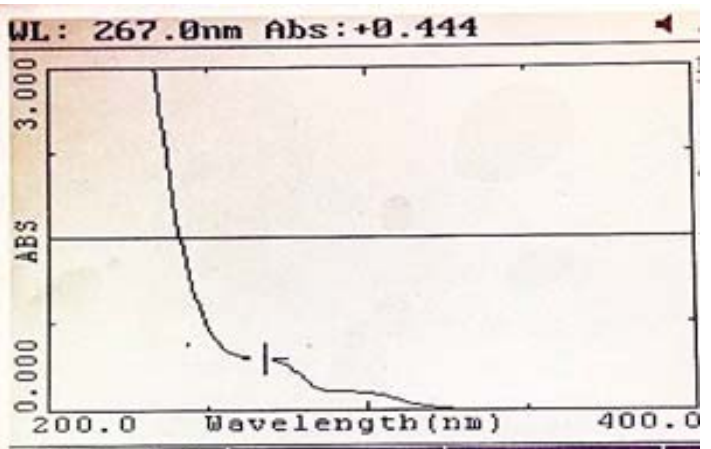

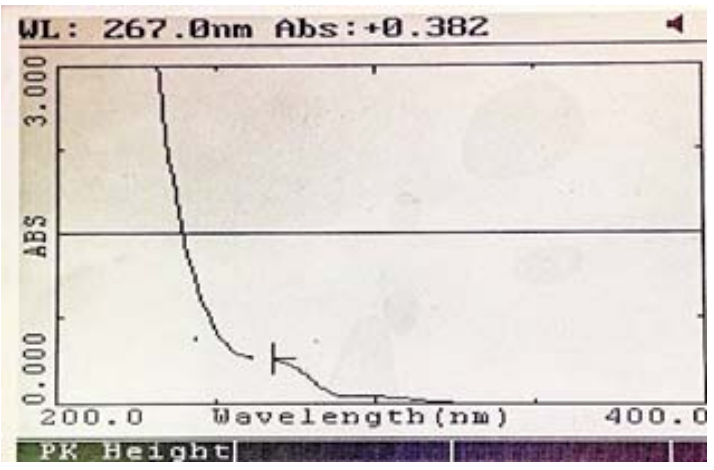

Fig. 5. 'Air Pump' aspirin absorbance decreasing from 0.448 to 0.382 at $267 \mathrm{~nm}$

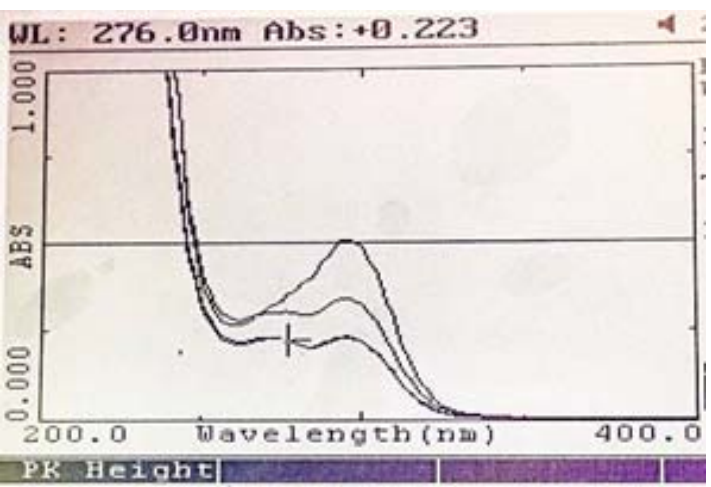

Fig. 6. Graphs showing the transformation of aspirin (left peak) into salicylic acid (right peak) in different temperature ranges during 20 minutes

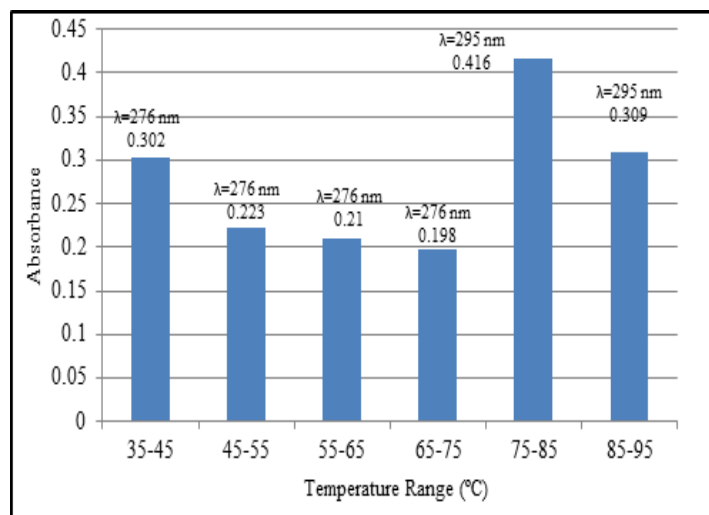

Fig. 7. Aerial Oxidation under the Influence of Increasing Temperatures 
Table. 3: Percentage Reduction via Ozonation

\begin{tabular}{cc}
\hline Sample Number & $\begin{array}{c}\text { Percentage } \\
\text { Degradation (\%) }\end{array}$ \\
\hline 1 & 0.619 \\
2 & 2.303 \\
3 & 2.201 \\
4 & 7.143 \\
\hline
\end{tabular}

as the photocatalyst. The weight of $\mathrm{TiO}_{2}$ and $\mathrm{pH}$ of the medium were optimized. $0.4 \mathrm{~g}$ of titanium dioxide catalyst was introduced to the solution and was allowed to settle. The $\mathrm{pH}$ of the sample was altered to 4 . The beaker was kept in open sunlight for $4-5 \mathrm{~h}$ and the sample was analyzed for changes on an hourly basis. Fig.9 \& Fig.10 summarized the observation.

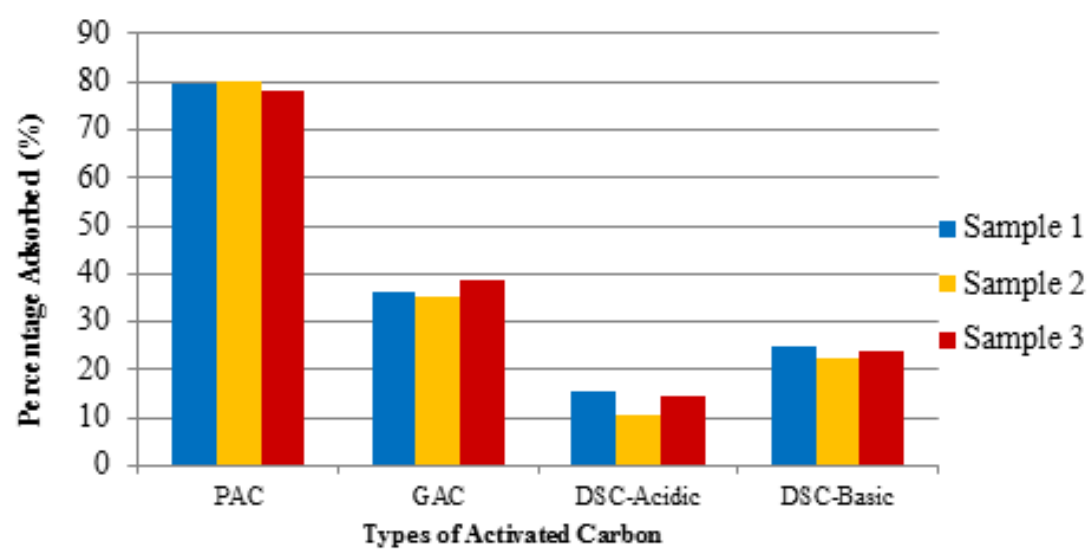

Fig. 8. Comparative Results showing Optimal Percentage Change obtained by different Carbons

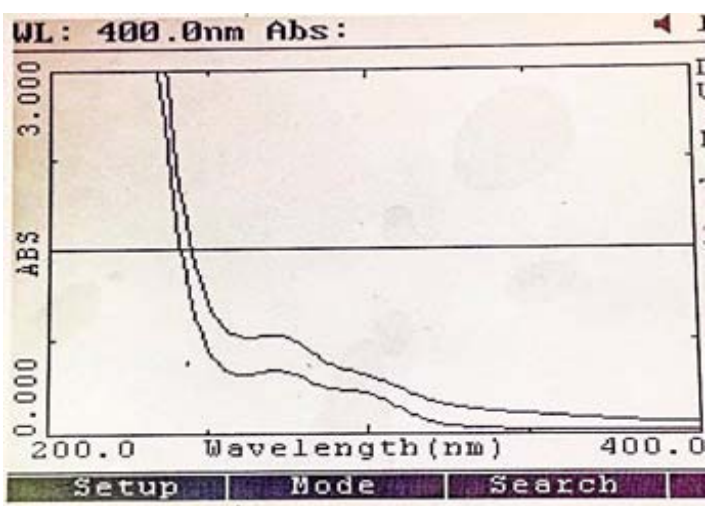

Fig. 9. Graph depicting a decrease in acetylsalicylic acid by $\mathbf{3 8 . 1 8 \%}$ (upper peak to lower peak)

RESULTS

For aeration, under ambient conditions aspirin took nearly 4 days to completely transform into salicylic acid. There is a gradual shift in the shoulder from acetylsalicylic acid to salicylic acid; i.e. from $\lambda=267 \mathrm{~nm}$ (represents acetylsalicylic acid) to $\lambda=295 \mathrm{~nm}$ (represents salicylic acid). After day 4, there is no longer a peak in the wavelength range of $267 \mathrm{~nm}$. There is only a single peak at $\lambda=295 \mathrm{~nm}$

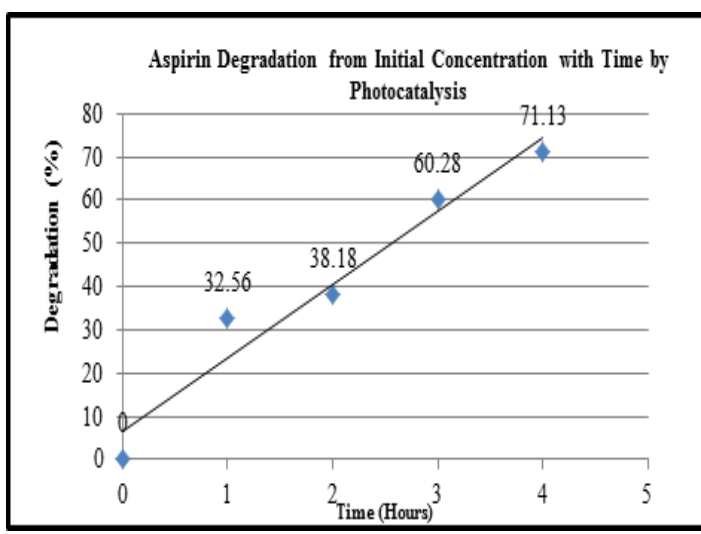

Fig.10. Graph showing Aspirin's Degradation in 4 hours

implying complete conversion of acetylsalicylic acid into salicylic acid. In 4 days, acetylsalicylic acid had almost completely transformed into salicylic acid. Acetylsalicylic acid began to transform into salicylic acid during a span of $8 \mathrm{~h}$ when air was bubbled through the solution using an air pump. In a span of $8 \mathrm{~h}$, aeration treatment could reduce/modify the amount of acetylsalicylic acid present in the sample by approximately $15 \%$. In practical situations, aeration is performed for approximately 6 hours. 
This study shows that acetylsalicylic acid would be relatively stable or undergo minimal transformation into salicylic acid during that time period. Under the influence of temperature, in samples 1-4, the UV-Spectrophotometric analysis showed a gradual shift in the wavelength from acetylsalicylic acid to salicylic acid. The wavelength shifted from $\lambda=267$ $\mathrm{nm}$ (at room temperature) to $\lambda=276 \mathrm{~nm}$ when subjected to increased temperatures (between $35-65^{\circ} \mathrm{C}$ ). The peak of acetylsalicylic acid began to decrease and that of salicylic acid began to increase. In samples 5 and 6 , there was no longer a peak for acetylsalicylic acid. Only one peak remained which corresponded to salicylic acid, i.e, $\lambda_{\max }=295 \mathrm{~nm}$. The $6^{\text {th }}$ sample shows a decrease in the absorbance from the $5^{\text {th }}$ by approximately 0.1 , indicating that salicylic acid was beginning to decompose /breakdown at that temperature. A further increase in temperature would lead to complete decomposition of salicylic acid.

The results obtained by ozonation showed change of the contaminant drug by $2-7 \%$. The pale yellow color formed indicates the occurrence of a reaction producing an aromatic compound (possibly quinine) which absorbs in the same wavelength range as that of aspirin and dosage. The increase in the adsorbent dose might cause aggregation of adsorbent, and consequently, the available adsorption sites might decrease. Therefore, it might be possible that adsorption capacity decreases as adsorbent dosage increases. Maintaining constant parameters of $\mathrm{pH}$, time and weight of carbons used, the optimal results have been summarized in Figure.8.

Over a span of four to five hours, the photocatalysis treatment with titanium dioxide could degrade the drug by $71 \%$. This proves that $\mathrm{TiO}_{2-}$ is a reliable substance for photocatalysis in the removal/ modification of acetylsalicylic acid.

\section{DISCUSSION}

Theoretically, aeration alone was expected to show substantial results. However, experimentally, it was not so. Aerial oxidation under ambient conditions managed to transform aspirin into salicylic acid over a period of 4-5 days. Aeration using a pump to pass air directly into the solution helped transform the aspirin into salicylic acid by $15 \%$ in a span of $8 \mathrm{~h}$. Aerial oxidation required the presence of high temperatures for complete conversion of the aspirin to occur. This worked best at a temperature range of $75-85^{\circ} \mathrm{C}$ where

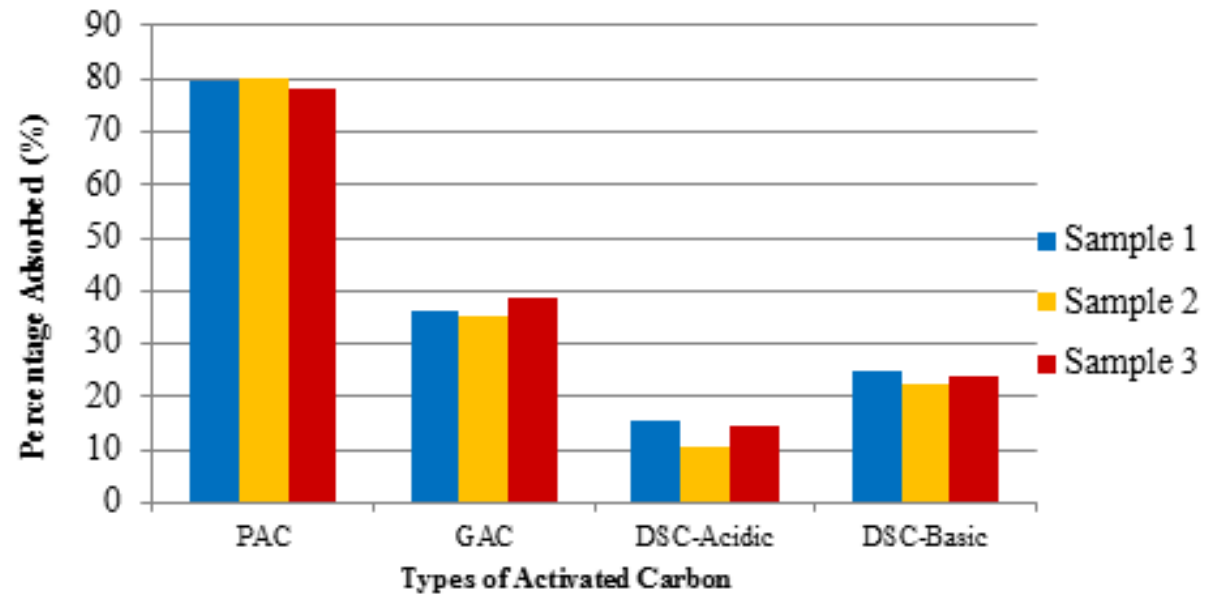

accommodates numerically whatever change/ reduction of aspirin that had taken place. This implies that Ozonation is not a useful method for treating Acetylsalicylic acid contaminants in water. $0.1 \mathrm{~g}$ of PAC showed a lesser adsorption value than $0.05 \mathrm{~g}$ because the adsorptive capacity of the adsorbent available was not fully utilized at a higher adsorbent dosage in comparison to lower adsorbent acetylsalicylic acid was completely transformed into salicylic acid within $20 \mathrm{~min}$. Further treatment methods needed to be explored in order to ensure the reduction of the content of aspirin in water.

Ozonation did not prove useful as there were reactions occurring that caused the production of other aromatic compounds like quinone which 
absorb in the same wavelength range as aspirin and hence the change in aspirin could not be monitored and the possible reduction in aspirin could not be estimated.

Adsorption was considered as the next treatment method and showed encouraging results. In terms of the best type of carbon, powdered activated carbon showed the best results with an adsorptive capacity of approximately $80 \%$. Since adsorption is an interaction of charges, this is a useful method as the charges on the adsorbent and adsorbate could be manipulated to enhance adsorptive capacity. If the surface of the adsorbent, activated carbon is neutral or near basic in nature, that is, if it has surface neutral or negative charges, then maximum adsorption would be obtained if the aspirin solution medium is buffered to be acidic and maintained at $\mathrm{pH} 4$ (so that positive ions dominate) which is the optimal $\mathrm{pH}$ for aspirin adsorption on this adsorbent.

If the surface of the carbon adsorbent has positive charges, it is best to not use a buffer so that the negative ions released by aspirin could dominate and enhance electrostatic interactions.

Photocatalysis of acetylsalicylic acid using Titanium dioxide showed that it is possible to degrade aspirin into salicylic acid which is further mineralizable. The highest photocatalytic activity of titanium dioxide is achieved in an acidic medium of $\mathrm{pH} 4$ for aspirin. There is substantial breakdown of the drug into salicylic acid in a short span of time; that is, four to five hours.

\section{CONCLUSION}

This comparative study of removal technologies for aspirin shows that adsorption using powdered activated carbon at its optimal conditions as mentioned, and photocatalysis using titanium dioxide in an acidic medium are the most effective methods amongst the rest of the processes practiced. These treatment technologies can be applied to other pharmaceutical compounds that share similar chemical characteristics and properties as that of acetylsalicylic acid.

The significance of this study lies in the cost-effectiveness and simplicity in the methodology of these treatment processes. Activated carbon is an easily available and immensely reliable adsorbent for the adsorption of chemicals like aspirin. Titanium dioxide photocatalysis is a stateof-the-art pharmaceutical wastewater treatment methodology and is already in use as a promising treatment technology for water purification. Substantial results can be obtained on an industrial level when these processes are directed towards the treatment of pharmaceutical pollutants in water.

\section{REFERENCES}

1. Heberer, T. Toxicology Letters. 2002, 131, 517

2. Benotti, M.J.; Trenholm, R.A.; Vanderford, B.J. Holady, J.C.; Stanford, B.D.; Snyder, S.A Environ. Sci. Technol. 2009, 43, 597-603

3. Heberer, T.; Reddersen, K.; Mechlinski, A Water Sci.Technol. 2002, 46, 81-88

4. Arcand Hoy, L.D.; Nimrod, A.C.; Benson, W.H. Int. J. Toxicol. 1998, 17, 139-158

5. Seehusen, D. A.; Edwards, J. J. Am. Board. Fam. Pract. 2002, 19, 542-547
6. Zhang, Y.; Geiben, S.; Gal, C. Chemosphere. 2008, 73, 1151-1161

7. Ternes, T.A.; Meisenheimer, M.; McDowell, D.; Sacher, F.; Brauch, H.J.; Haist-Gulde, B.; Preuss, G.; Wilme, U.; Zulei-Seibert, N. Environ. Sci. Technol. 2002, 36, 3855-3863

8. Hashimoto, K.; Irie, H.; Fujishima, A. Jpn. J. Appl. Phys. 2005, 44, 2-6

9. Jones, O.A.H.; Voulvoulis, N.; Lester, J.N. Crit Rev. Environ. Sci. Technol. 2005, 35, 401-427 